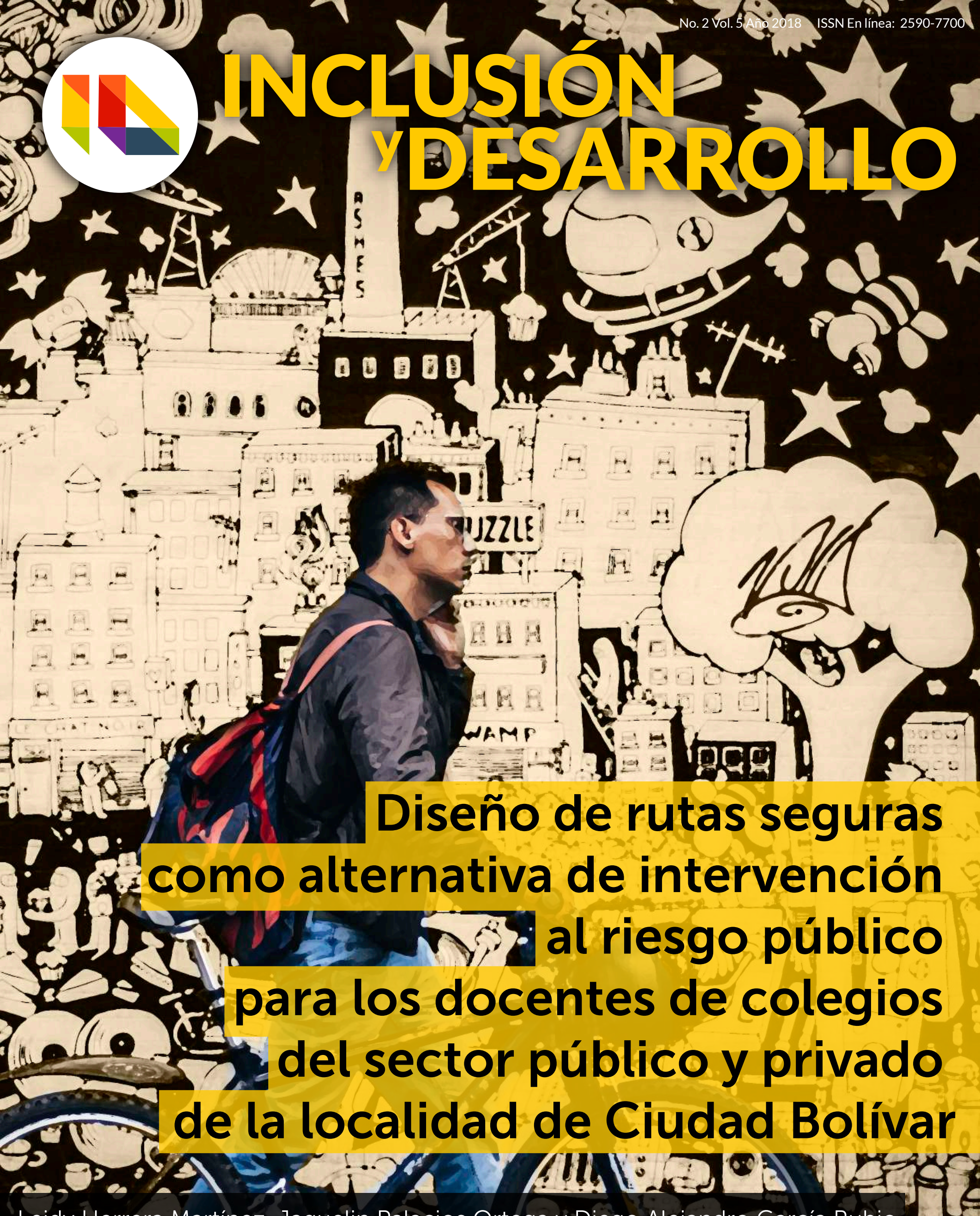




\section{DE INTERVENCIÓN AL RIESGO PÚBLICO PARA LOS DOCENTES \\ DE COLEGIOS DEL SECTOR PÚBLICO Y PRIVADO DE LA LOCALIDAD DE CIUDAD BOLÍvAR}

\section{DESIGN OF SAFE ROUTES AS AN ALTERNATIVE OF INTERVENTION TO PUBLIC RISK FOR THE TEACHERS OF PUBLIC AND PRIVATE SECTOR SCHOOLS OF THE GIUDAD BOLÍVAR}

$$
\begin{array}{r}
\text { Leidy Herrera Martínez } \\
\text { Iherrerama3@uniminuto.edu.co } \\
\text { Corporación Universitaria Minuto de Dios-UNIMINUTO } \\
\text { Bogotá-Colombia } \\
\text { Jaquelin Palacios Ortega } \\
\text { jpalacioso1@uniminuto.edu.co } \\
\text { Corporación Universitaria Minuto de Dios-UNIMINUTO } \\
\text { Bogotá-Colombia }
\end{array}
$$

Diego Alejandro García Rubio dgarciarub2@uniminuto.edu.co Corporación Universitaria Minuto de Dios-UNIMINUTO Bogotá-Colombia

\section{Resumen}

Objetivo. Analizar las diversas problemáticas del riesgo público, al cual están expuestos los docentes en los centros educativos: Colegio el Minuto de Buenos Aires y Colegio Confraternidad de San Fernando en la localidad de Ciudad Bolívar de la ciudad de Bogotá. Metodología. Análisis cualitativo implementado por medio de aplicación de encuestas y entrevistas con enfoque descriptivo y participativo.

Resultados. Como resultado a través del trabajo en campo se analiza la percepción de inseguridad que presentan los profesores y finalmente se plasma la alternativa de prevención y mitigación respectiva, generando alternativas en función de los objetivos planteados que ayuden a su seguridad personal, fomentando el desarrollo social en las instituciones educativas para el bienestar de los docentes.

Originalidad. Se expone el diseño de rutas seguras como medida de intervención para disminuir los diferentes factores de riesgo público a los cuales se encuentran expuestos docentes en sus desplazamientos diarios a sus lugares de trabajo y viceversa.

Palabras clave: representaciones sociales, trabajo infantil, política pública de trabajo infantil. 


\section{Abstract}

Objective. To analyze the different problems related to public risk, where teachers are exposed in educational centers, such as: Minuto de Buenos Aires School and Confraternidad de San Fernando School located in Ciudad Bolívar in the city of Bogotá. Methodology. The qualitative analysis was implemented through the application of surveys and interviews with a descriptive and participative approach.

Results. As a result through the fieldwork, he perception of insecurity that the professors present is analyzed, and finally the respective prevention and mitigation alternative are formulated, generating alternatives based on the proposed objectives that help their personal security, fostering social development in educational institutions for the welfare of teachers.

Originality. The design of safe routes is exposed as an intervention measure to reduce the different public risk factors, where teachers are exposed in their daily commutes to their places of work and vice versa.

Keywords: insecurity, theft, threats, public risk, teacher and safe routes. 


\section{INTRODUCCIÓN}

Cl país en los últimos años ha estado inmerso - en una serie de reformas que buscan el desarrollo social, económico y educativo. Por ello el desarrollo social implica una evolución de cambio positivo, por lo cual lo que se quiere lograr en el campo de las instituciones educativas es principalmente el desarrollo en el bienestar social, en el caso de los docentes en base a su seguridad personal. (Rodríguez H, 2012).

Por otro lado, la violencia es uno de los principales problemas para los docentes en las aulas evidenciándose este en los aspectos físicos o psicológicos, o en su defecto las dos al mismo tiempo; si bien es cierto que se trata de un reflejo de la violencia existente en la sociedad actual, representa un problema cada vez más preocupante, ya que la inseguridad afecta al profesorado de manera constante, particularmente en estas dos instituciones educativas y que se pudo evidenciar en las encuestas y entrevistas realizadas.

Entiéndase como Riesgo Público el daño potencial que, sobre la población y sus bienes, la infraestructura, el ambiente y la economía pública y privada, pueda causarse por la ocurrencia de amenazas de origen natural, socio-natural o antrópico no intencional, que se extiende más allá de los espacios privados o actividades particulares de las personas y organizaciones y que por su magnitud, velocidad y contingencia hace necesario un proceso de gestión que involucre al Estado y a la sociedad. (DECRETO 423 DE 2006, art. 3).

Por lo anterior el riesgo público es un componente importante en lo que compete a la hora de brindar seguridad a los trabajadores, ya que por los diferentes ambientes, externo e internos de tensión que se manejan hoy en día son muy diversos, conlleva a que existan conductas violentas hacia los trabajadores viéndose afectadas directa o indirectamente por actos de inseguridad externa a sus instalaciones de trabajo, ya sea siendo víctimas de amenazas, atracos a mano armada, extorsiones, microtráfico, entre otros; por ello el interés de dar a conocer las diferentes medidas y alternativas de seguridad que pueden adoptar la planta de docentes de los dos colegios de ciudad Bolívar para su prevención ante el riesgo público.

Según el estudio de Gómez (2010) describe que: "En los docentes existen situaciones de miedo, angustia o temor; desconcierto e incertidumbre; inseguridad y desconfianza; frustración, impotencia y desesperanza; tristeza y otras emociones derivadas de la situación de violencia"; lo cual da a entender por qué en la

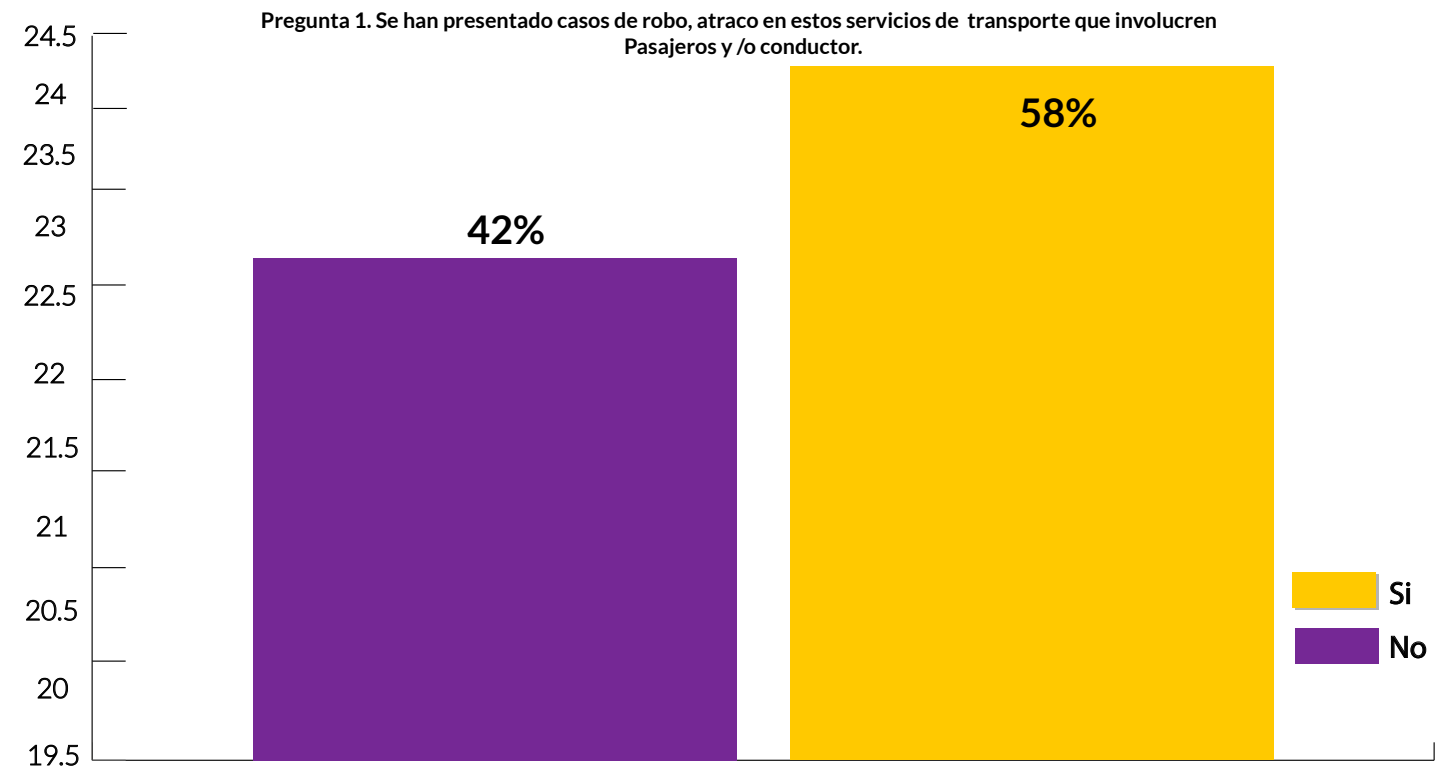

Figura 1. (Resultado de encuesta de Existencia de factores de riesgo público en desplazamientos; fuente propia 2017). 
mayoría de casos no se realiza la divulgación a la comunidad y mucho menos a las autoridades competentes de estos casos ya que no es fácil afrontar los efectos que esto conlleve y que en su mayoría pueden ser negativos.

La inseguridad surge y se define en la actualidad como un fenómeno y problema social en países que poseen un diverso nivel de desarrollo económico, múltiples rasgos culturales y regímenes políticos de distinto signo, no pudiéndose establecer, por tanto, distinciones simplistas para caracterizar factores asociados a su incremento y formas de expresión. La Amenaza es considerada un peligro latente, en donde puede que ocurra un evento que cause afectaciones en las personas, ya sea por origen natural, o causado por la acción humana de manera accidental o planeada, dependiendo de su severidad puede causar pérdida de vidas, lesiones o impactos en la salud, así como también daños en los bienes, la infraestructura, los medios de sustento, la prestación de servicios y los recursos ambientales. (González Plasencia, Álvarez, \& Arce, 2009)

Es por ello que ante tal percepción de inseguridad que viven los docentes hoy en día y en especial en estos dos colegios de Ciudad Bolívar, se generan estos posibles caminos de mejora por medio de alternativas de seguridad y por lo que se abordó a la pregunta, ¿Qué alternativa de seguridad se debe tener en cuenta como prevención ante un factor de riesgo público?

El proyecto de investigación busca identificar las diferentes problemáticas vinculadas al riesgo público: inseguridad, amenazas, hurtos, extorsión, actos violentos y vandálicos, microtráfico, entre otros, a los cuales están expuestos los docentes de los centros educativos tanto del sector público como del sector privado en la localidad de ciudad bolívar en Bogotá, quienes a través de su percepción y experiencias propias permitirán la elaboración del diseño de rutas seguras como prevención ante el riesgo público.

\section{ESTADO DEL ARTE Y ANTECEDENTES PRÁCTICOS}

Este artículo se elaboró con base a la revisión de información primaria por medio de aplicación de encuestas y entrevistas y secundaria, por medio de noticias, artículo y revistas, en donde se revisan los temas más relevantes sobre el riesgo público con enfoque hacia los docentes que permita identificar a grosso modo la atención investigativa y analítica que está teniendo en los últimos años recientes el tema de riesgo público en docentes. Mediante un trabajo de revisión exhaustiva de la bibliografía disponible, se concentró en los siguientes documentos que resultaron ser puntos de confluencia conceptual

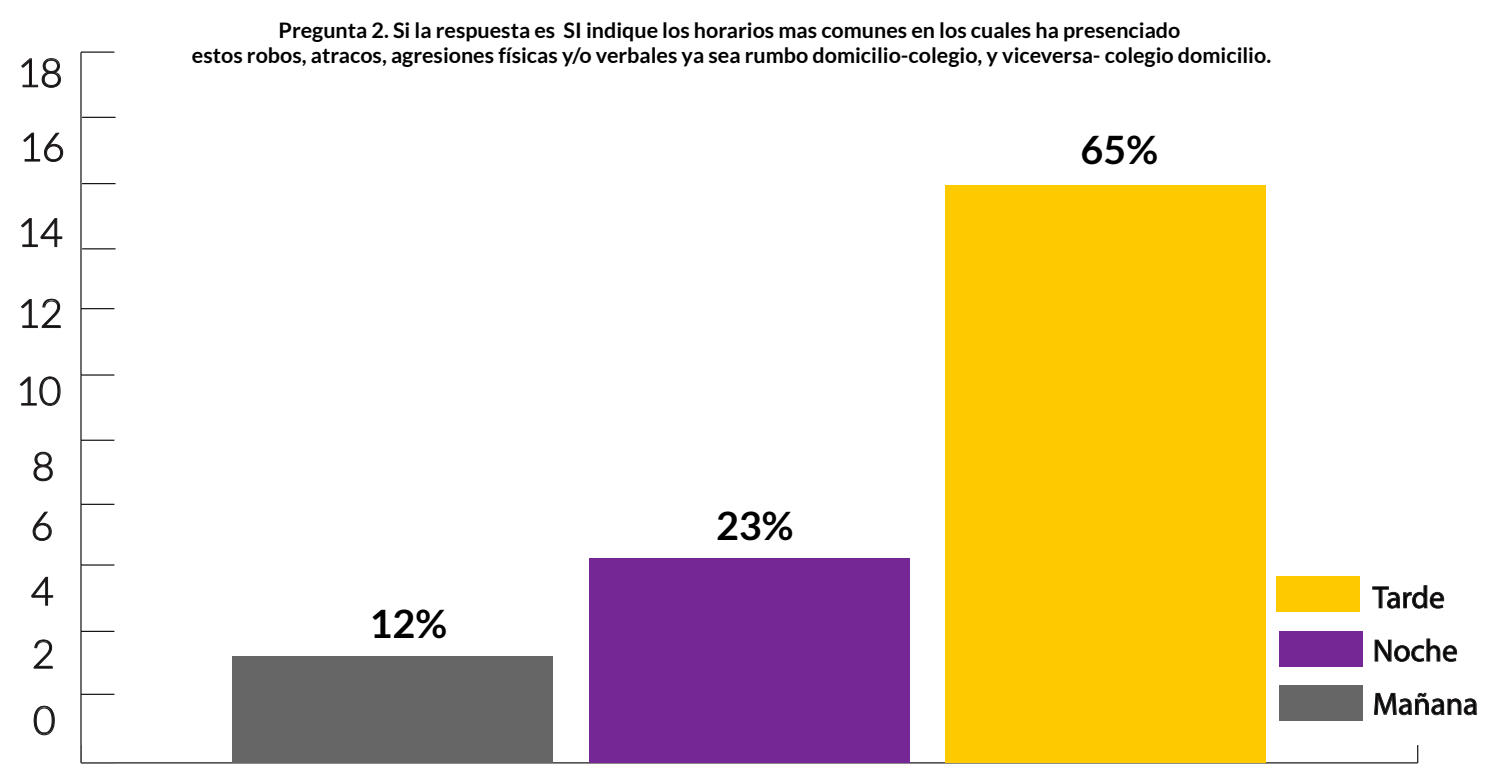

Figura 2. (Resultado de encuesta de Jornadas Con presencia De Factor de Riesgo Público; fuente propia 2017). 
e investigativa de la situación desde diversas perspectivas.

En primer lugar, se presenta como antecedente el siguiente artículo "Influencia de la violencia en el medio escolar y en sus docentes: estudio en una localidad de Bogotá Colombia." Por estudiantes de médicos de la Universidad Javeriana. (Rev. Colomb. Psiquiat., vol. 39 / No. 1 / 2010), el cual tiene como objetivo conocer la influencia de violencia y de pandillas en el que hacer del docente, en donde se obtiene como resultados situaciones de miedo, angustia, e inseguridad, estudio aplicado en el colegio de san Cristóbal de la localidad de Ciudad Bolívar.

Por otro lado, se encontró una noticia publicada por el periódico el tiempo en donde señala Según cifras divulgadas por el Ministerio de Educación que durante los años 2015-2016, se amenazaron en Colombia 605 docentes de los cuales Bogotá ocupa el primer puesto con un total de 223 casos. (MEN. 2016).

Y por último se encuentra un estudio realizado por la secretaria de Bogotá denominado "Plan integral de seguridad convivencia ciudadana y justicia 2017-2020", en donde señala las características de los fenómenos de seguridad, convivencia y justica de localidad de Ciudad Bolívar más relevantes, por lo cual según el Sistema de Información Estadístico,
Delincuencial, Contravencional y Operativo (SIEDCO) de la DIJIN de la Policía Nacional, señala los delitos que más afectaron a la localidad de Ciudad Bolívar durante 2016 como el homicidio, las lesiones personales y el hurto a personas.

\section{MARCO TEÓRICO Y REFERENTES CONCEPTUALES}

El proyecto de investigación busca identificar las diferentes problemáticas vinculadas al riesgo público: inseguridad, amenazas, hurtos, extorsión, actos violentos y vandálicos, microtráfico, entre otros, a los cuales están expuestos los docentes en sus desplazamientos de los centros educativos tanto del sector público como del sector privado en la localidad de ciudad bolívar en Bogotá, quienes a través de su percepción y experiencias propias permiten el desarrollo de los objetivos y la implementación de estrategias dentro de la investigación. Recordemos que el riesgo público son todos aquellos aspectos que se viven en espacios públicos y que pueden poner en riesgo la vida y la integridad física de las personas. Por lo general están relacionados con tránsito y violencia (Gómez et al, Restrepo, Padilla, Rodríguez, Guzmán, Mejía, 2010).

Por consiguiente, la Inseguridad ciudadana surge y se define en la actualidad como un fenómeno y problema social en países que poseen un

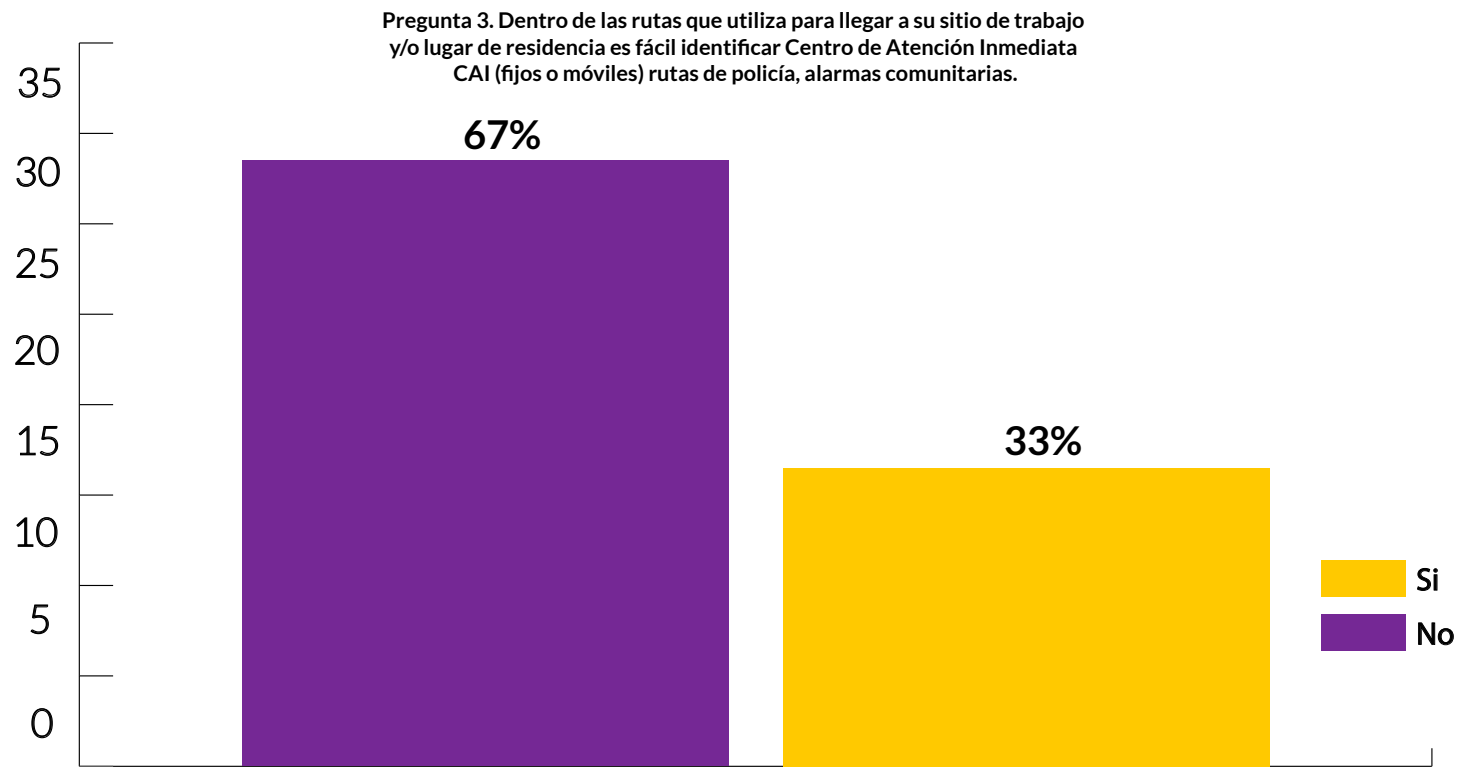

Figura 3. (Resultado de encuesta de Identificación de CAI, rutas y alarmas; fuente propia 2017). 


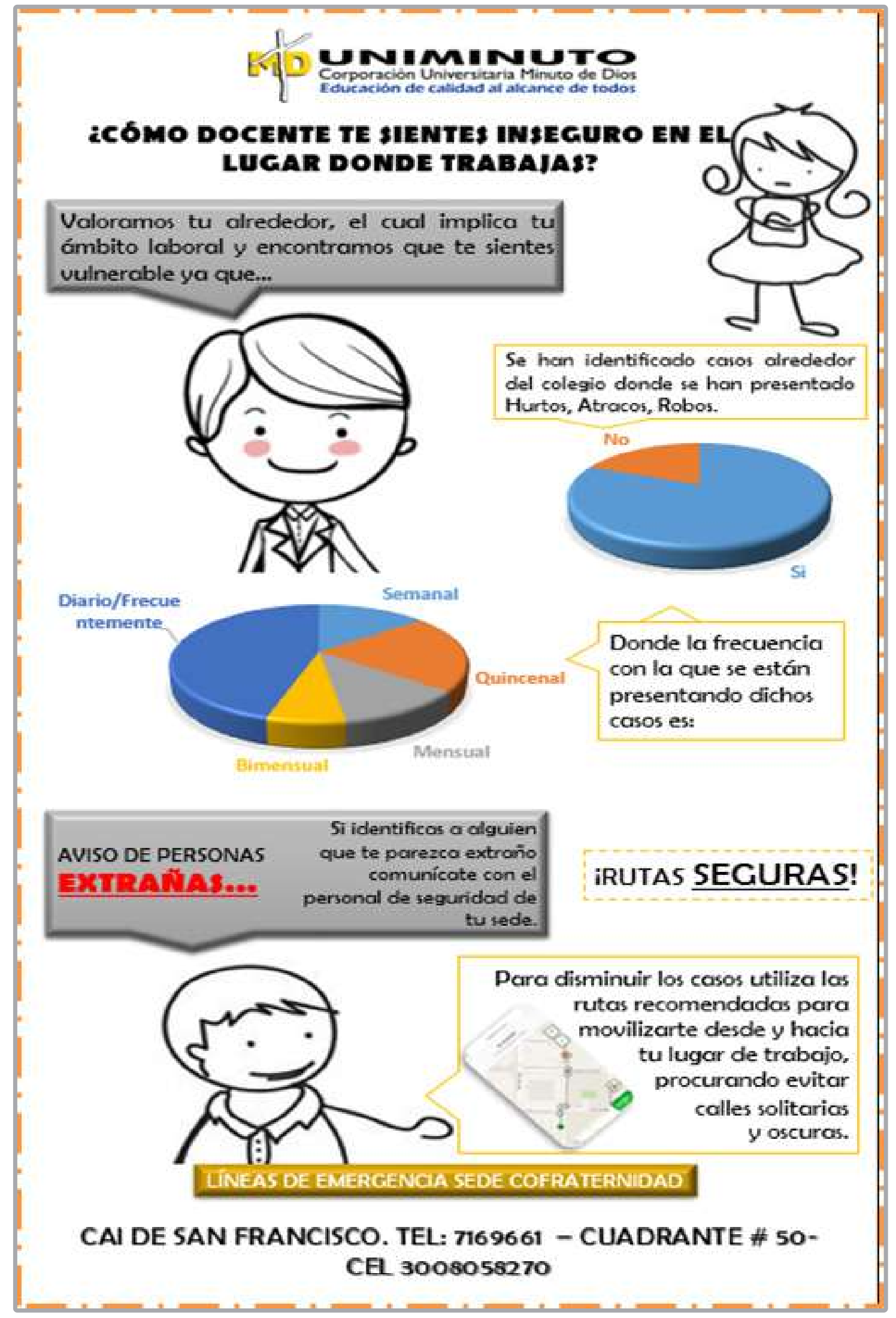

Figura 4. (Hablador: Uso De Rutas Seguras; fuente propia 2017). 
diverso nivel de desarrollo económico, múltiples rasgos culturales y regímenes políticos de distinto signo, no pudiéndose establecer, por tanto, distinciones simplistas para caracterizar factores asociados a su incremento y formas de expresión. La Amenaza es considerado un peligro latente, en donde puede que ocurra un evento que cause afectaciones en las personas, ya sea por origen natural, o causado por la acción humana de manera accidental o planeada, dependiendo de su severidad puede causar pérdida de vidas, lesiones o impactos en la salud, así como también daños en los bienes, la infraestructura, los medios de sustento, la prestación de servicios y los recursos ambientales. (Garzón L, 2015).

\section{Metodología}

El desarrollo de esta investigación se lleva a cabo con base a un estudio descriptivo (Cleroux, 1993), en el cual busca especificar propiedades y características importantes de cualquier fenómeno que se analice y describa las tendencias de un grupo o población. Con un enfoque cualitativo el cual se utiliza para la recolección y análisis de los datos para afinar las preguntas de investigación o revelar nuevas interrogantes en el proceso e interpretación. (Escamilla, 2018)

Por lo cual, del trabajo de campo realizado por medio de rastreo de datos, aplicación de encuestas, entrevistas, recopilación de datos históricos y análisis del entorno, se conocieron los diferentes factores de riesgo público, como situaciones de inseguridad, amenazas, robos, micro tráfico que evidencian en sus desplazamientos por los cuales se enfrentan diariamente los docentes de los colegios Confraternidad del sector privado y el Minuto de Buenos Aires del sector público de la localidad de Ciudad Bolívar, con el fin de la elaboración de diseño de rutas seguras, como se describe en las siguientes fases de investigación de campo.

Fase 1: Recolección de información: Esta fase se inicia con la búsqueda de información por medio de rastreo de datos referentes o que presentaran cercanía con el tema del riesgo público con enfoque hacia los docentes, por medio de

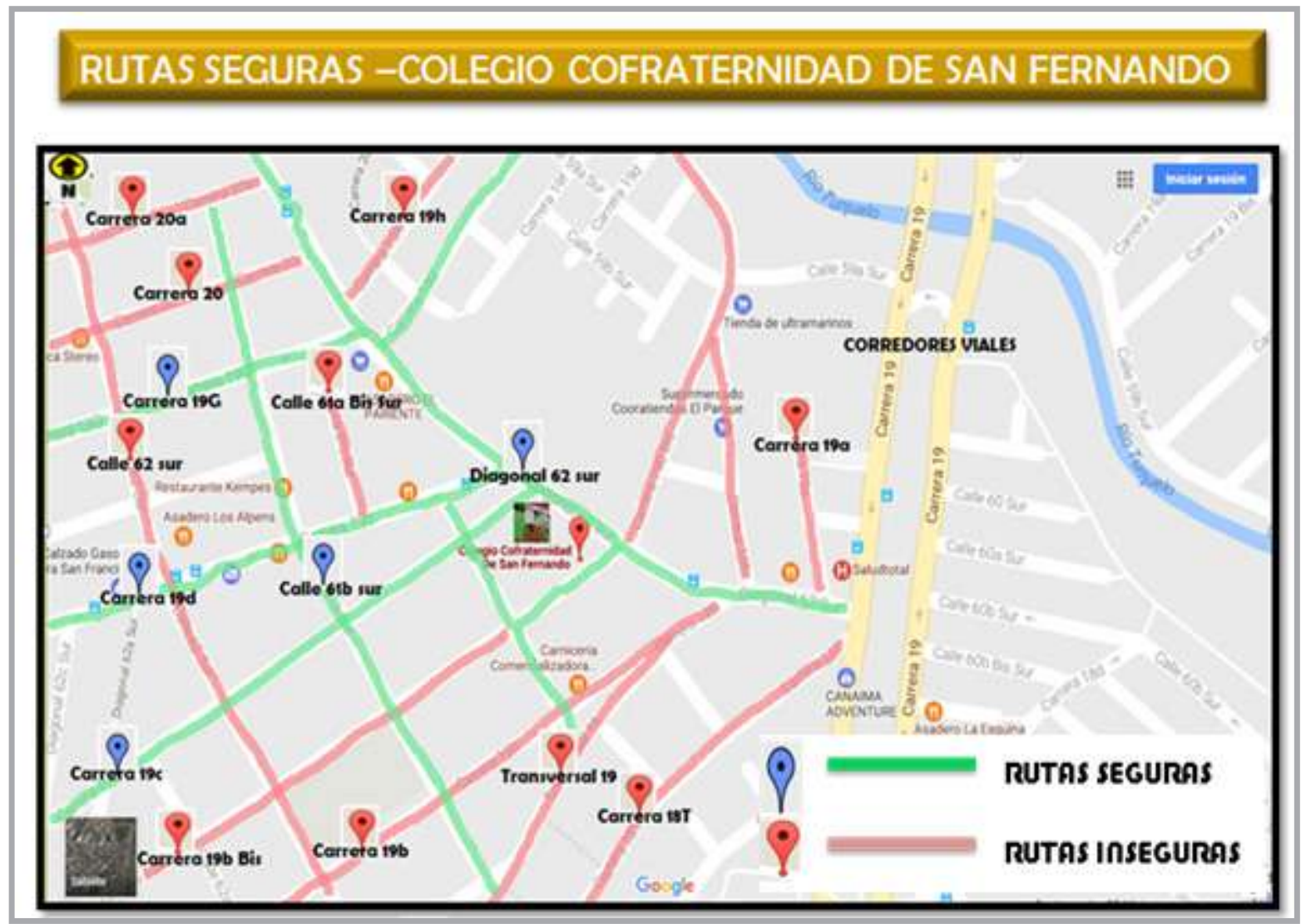

Figura 5. (Mapeo de identificación de rutas seguras e inseguras de la zona de San Francisco, colegio Cofraternidad de San Fernando (fuente propia 2017). 
fuentes como periódicos, noticias, artículos y libros; análisis histórico, como en el libro Entorno y posibilidades del ejercicio profesional y sindical de los docentes en Colombia. Educación y violencia, 45-61. (Acosta, 2015). Dicha información fue documentada en la elaboración de un cuadro Resumen Académico Educativo. De igual manera se realizó la búsqueda demográfica de las dos instituciones para con ellas realizar la superposición de planos y mapas, identificando en ellos en primera instancia las instituciones educativas intervenidas, rutas seguras y CAI cercanos.

Fase 2: Trabajo de campo: Intervención en los centros educativos por medio de la presentación del proyecto a desarrollar, y registro fotográfico del entorno, interacción con el personal de las instituciones (docentes, personal de seguridad), comunidad (habitantes de los alrededores) y funcionarios de centros de acción inmediata (cuadrantes y CAÍ) más cercanos a las instituciones, obtención de datos por medio de la aplicación de encuestas y entrevistas.

Fase 3: En el análisis de la información y la triangulación de los datos, se realizó la interpretación de los resultados, frente a las diferentes modalidades del riesgo público en la comunidad docente de los colegios de Ciudad Bolívar con el fin de evaluar el entorno

Fase 4: Definición de medidas de prevención y elaboración de diseño de rutas seguras por medio de mapeo e identificación de rutas seguras e inseguras y localización de CAI cercanos ante eventualidades de riesgo público.

\section{PerCePCIÓN DE INSEGURIDAD}

Es importante destacar el planteamiento de Jiménez (2015), en la publicación de la revista de Inclusión \& Desarrollo, en su artículo de "Educación, Investigación Y Desarrollo Social" en donde se nombra el artículo "Investigación, equidad y cohesión social" por la cual plantea que la educación se convierte en una herramienta importante para superar viejas y nuevas formas de desigualdad y exclusión social, a través del acceso equitativo a una educación de calidad, a lo largo y ancho del sistema educativo; es por ello que la educación, como instrumento de preparación de corto, medio y largo alcance, es una herramienta fundamental del desarrollo, del conocimiento y de la cohesión social. Por lo anterior, esta investigación se enlaza con el sector educativo ya que la población a estudio es su planta de docente la

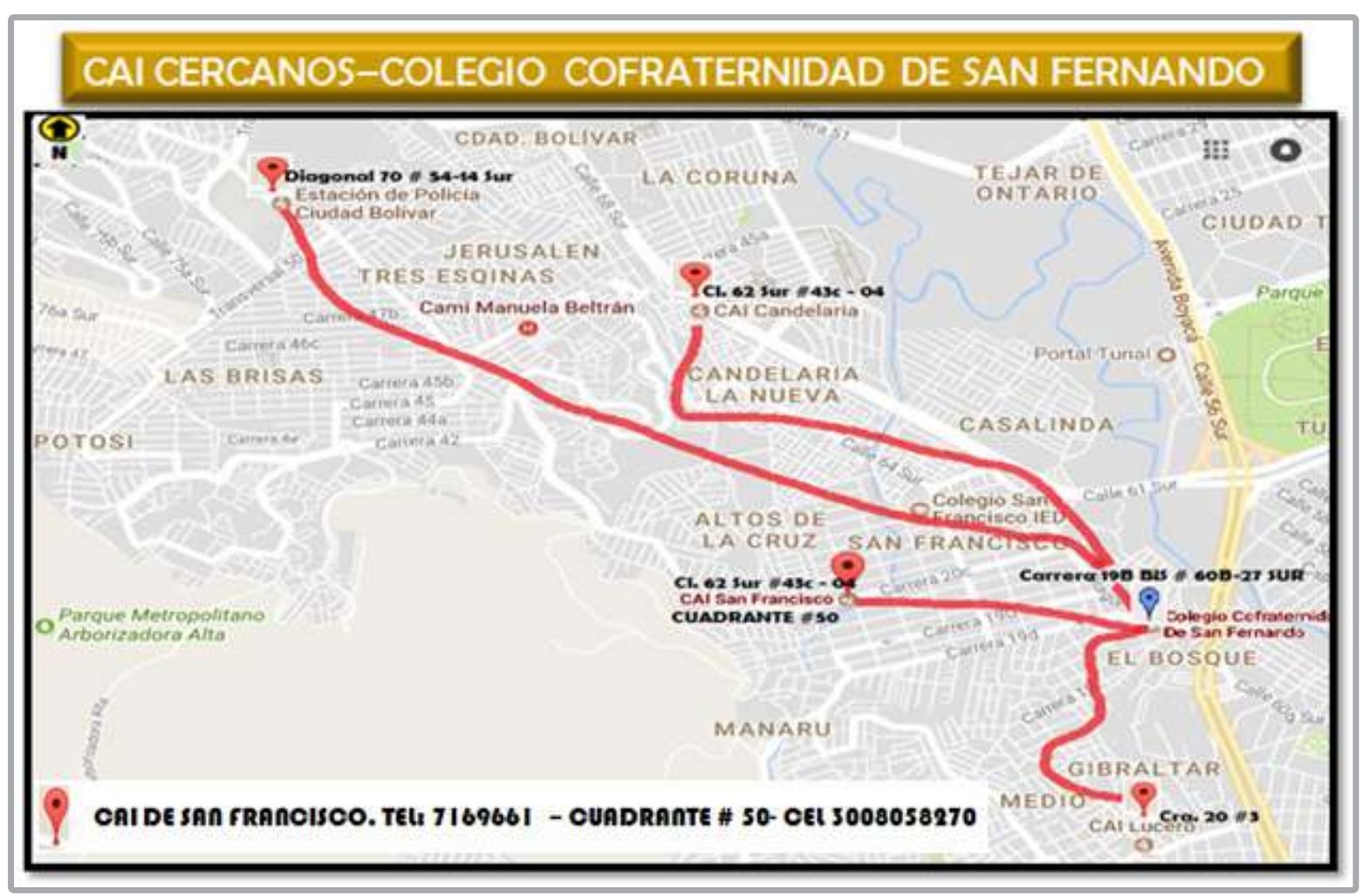

Figura 6. (Mapeo de rutas de Caí de policías cercanos al colegio de Cofraternidad de San Fernando; fuente propia 2017) 
cual hace parte de dicho sistema educativo, y lo que se busca es hacer visibles aquellas realidades más alejadas, como lo es la importancia del riesgo público, mostrando posibles caminos para la mejora, enfocado a la prevención y bienestar de sus trabajadores por medio de alternativas de seguridad.

Se dio alcance en las dos instituciones a un total de 45 docentes; por consiguiente, la percepción de inseguridad en los entornos de las Instituciones Educativas y durante el tránsito o recorrido para la asistencia de los docentes en la localidad, se encuentra ligado en un $80 \%$ de los encuestados en manifestar haber sido víctimas o conocer de manera directa e indirecta de situaciones de atracos, hurtos o robos con una recurrencia semanal, aunque no existe detalle de los momentos o los actores implicados, el temor es constante y manifiestan estar en situación de riesgo y ser víctimas de este flagelo.

En la figura 1 se muestra una de las preguntas realizadas en la encuesta que se llevó a cabo a los docentes en las dos instituciones la cual generaba la interrogante de si se han presentado casos de robo, atraco en estos servicios de transporte que involucren Pasajeros y /o conductor, con el fin de identificar si los docentes mientras se desplazan de sus hogares al lugar de trabajo y viceversa se sienten expuestos o han sido víctimas de acciones violentas a lo cual un 58\% de los encuestados; es decir, 26/45 docentes han evidenciados estos casos; eventos que no suelen ser reportados a las autoridades.

Luego de revisar la pregunta 20 de la encuesta (Figura 1) se e realiza la pregunta 21 (Figura 2) a los docentes donde si en la pregunta 20 respondieron SI, se les preguntaba con qué frecuencia han sido afectados por este tipo de afectaciones (robos, atracos, agresiones físicas y/o verbales ya sea rumbo domicilio-colegio, y viceversa- colegio domicilio) donde 17 de estos docentes que respondieron SI corroboran que la frecuencia con que se suelen presentar más estos casos es en el horario de la Tarde siendo ellos un $65 \%$, seguido de 6 docentes que consideran que es en la Noche en un $23 \%$ y por ultimo 3 docentes consideran que se efectúan estos casos en la mañana en un $12 \%$; lo que quiere decir, que los docentes se sienten amenazados y expuestos en la jornada de la tarde.

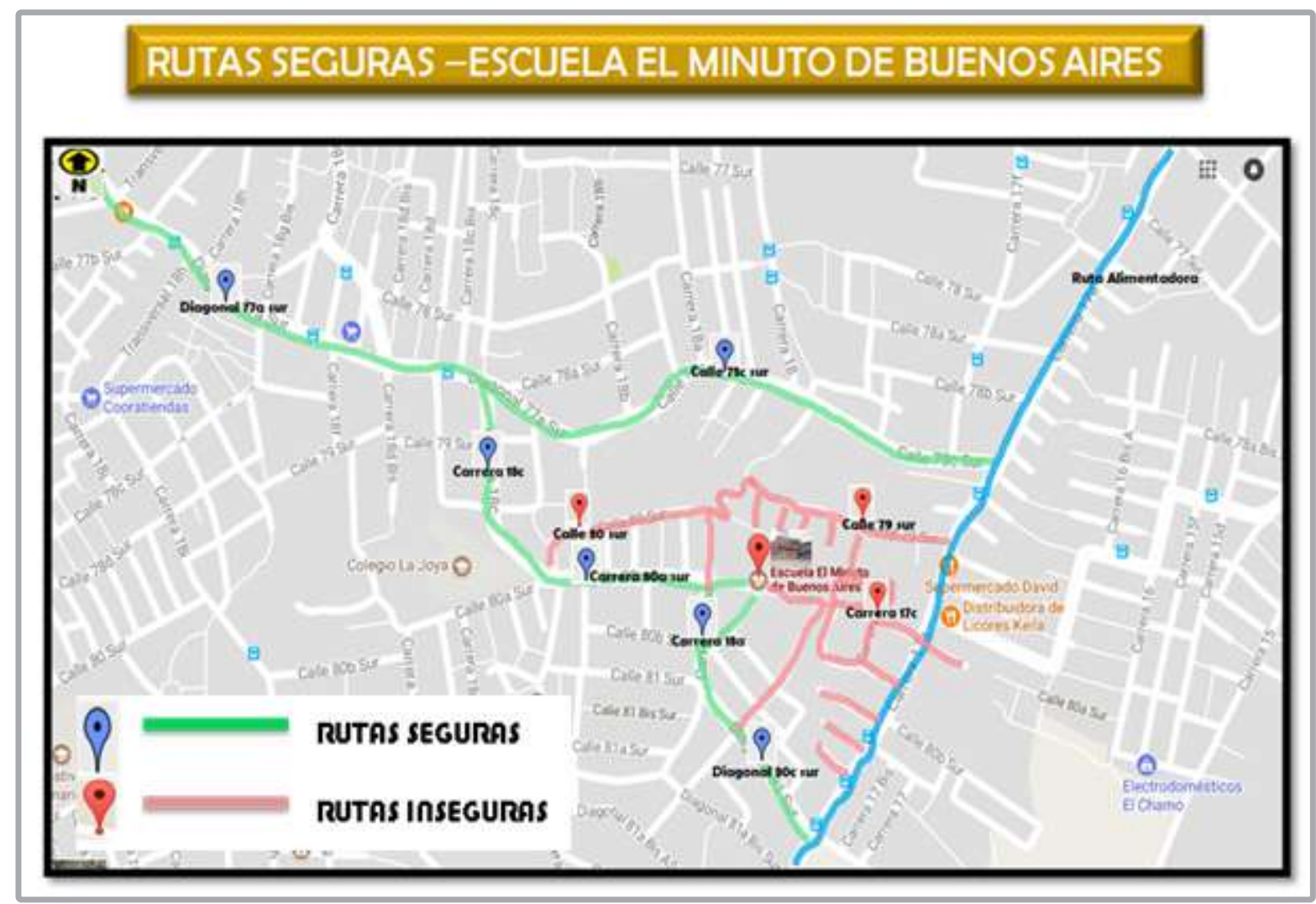

Figura 7. (Mapeo de identificación de rutas seguras e inseguras de la zona de San Joaquín, colegio el minuto de buenos aires; fuente propia 2017). 
Al encontrarse horarios específicos y determinar que en el recorrido que realizan los docentes se sienten vulnerables se les realiza la pregunta número 22 de la encuesta (Figura 3), en donde se evidencia; además que los docentes no conocen los puntos en los cuales pueden ayudarse como Centros de Atención Inmediata CAI o si de las autoridades realizan seguimiento continuo a los alrededores; donde un $67 \%$ de los encuestados; es decir, 30 docentes desconocen dentro de su ruta habitual para llegar a su lugar de trabajo si existen CAIS (fijos o móviles) rutas de policía o alarmas comunitarias; por ello otra alternativa que involucra las rutas seguras es el acercamiento y visualización clara por parte de esta comunidad, los sitios o puntos a los que pueden acudir en caso de sentirse amenazados.

\section{ANÁLISIS DE RESUltADOS}

El aporte que se pretende dar a la comunidad educativa y en especial a los colegios investigados es la prevención ante los factores de riesgo público que se evidencian en estas zonas donde quedan ubicados los colegios que fueron objeto de estudio, con enfoque hacia los docentes, contando con la participación de cada uno de ellos, los cuales se tuvieron como apoyo la policía de los cuadrantes de cada sector y los vigilantes de dichos colegios para identificación de rutas seguras e inseguras. A Continuación se evidencia el diseño de rutas seguras en las Figuras 4, 5, 6, 7 y 8, se inicia con una hablador donde se aclara la importancia de utilizar las rutas seguras como prevención ante riesgo público, se muestra los corredores viales de cada colegio, con la identificación de rutas seguras y rutas inseguras, y por otro lado el mapeo de los CAI cercanos a cada colegio en caso de que se necesiten refuerzos en alguna eventualidad de riesgo público, se denotan números de contacto y direcciones en cada uno de ellos.

Uno de los colegios intervenidos es el colegio Cofraternidad de San Fernando, colegio que se encuentra en un sector muy comercial y concurrido por ello se da a conocer las diferentes rutas que pueden tomar y las también las principales para que los docentes que laboran allí tengan alternativas y se puedan movilizar un poco más seguros.

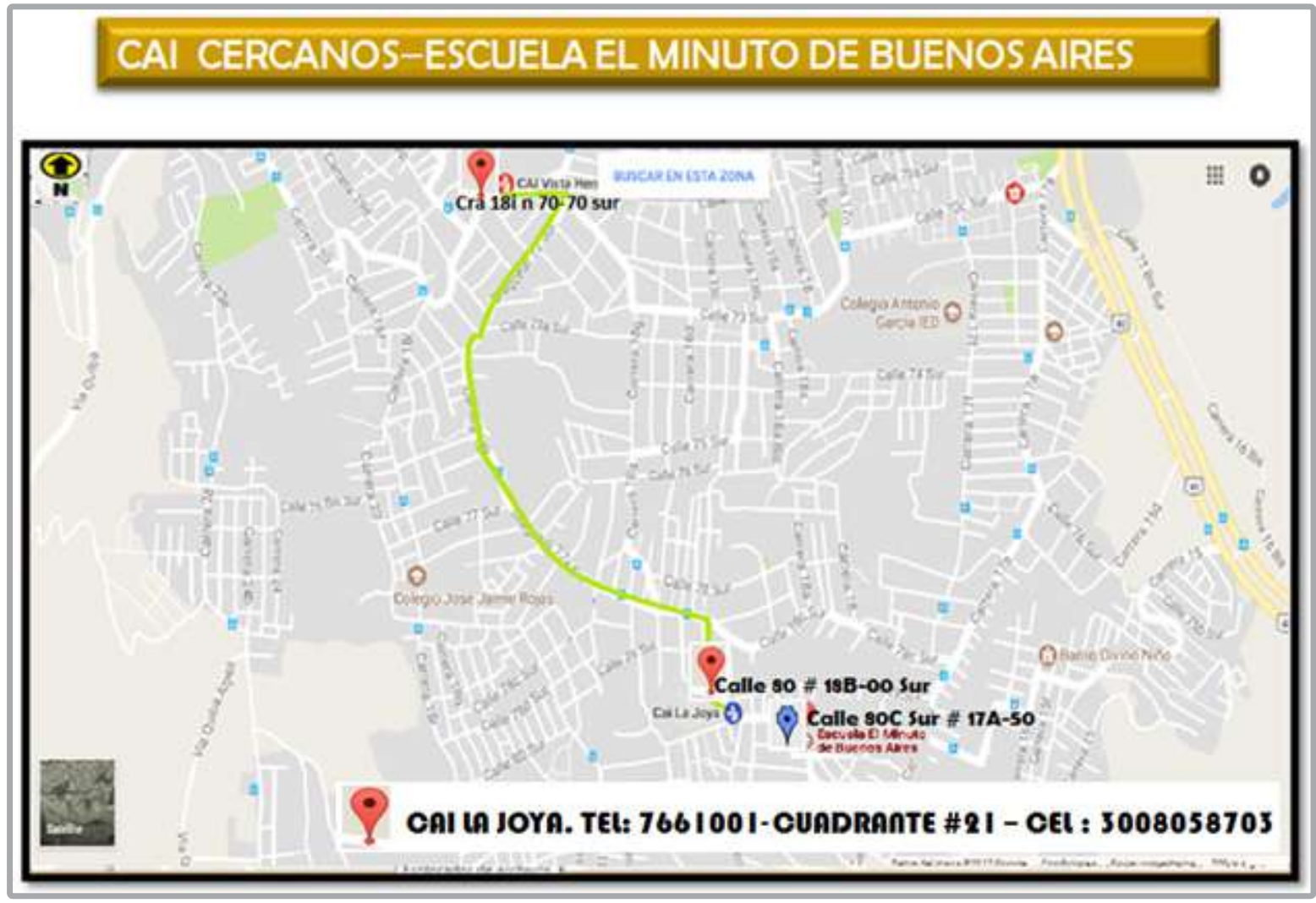

Figura 7. (Mapeo de rutas de Caí de policías cercanos al colegio de Minuto De Buenos Aires; fuente propia 2017). 
En la figura 6 del colegio Cofraternidad de San Francisco, se da a conocer los CAI cercanos a los que pueden tener acceso los docentes y en sí la comunidad en general para el reporte de casos o para estar enterados de donde quedan ubicados.

El segundo colegio es el colegio El Minuto de Buenos Aires el cual se encuentra en una zona más rural y menos comercial, también es una zona más alejada en comparación con el primer colegio y en donde los docentes desconocen las rutas más apropiadas para el transito seguro por ello se señalan las rutas más apropiadas para que esta comunidad de docentes pueda transitar.

Se realiza la ubicación del CAI disponible para esta comunidad y se evidencia que, aunque se encuentra retirado también existen medios de comunicación telefónica a los cuales las autoridades dan respuesta para realizar cualquier acción; sin embargo, es necesario que los docentes lo conozcan ya que, al quedar tan retirado, se pueden encontrar más expuestos y vulnerables.

\section{Conclusiones}

El riesgo público es un componente importante en lo que compete a la hora de buscar alternativas para disminuir la inseguridad a la que se ven expuestos los trabajadores, ya que por los diferentes ambientes externo e internos de tensión que se maneja hoy en día es muy diversa, por lo que conlleva a que existan conductas violentas hacia los trabajadores, viéndose afectados directa o indirectamente por actos de inseguridad externa a sus instalaciones de trabajo, ya sea siendo víctimas de amenazas, atracos a mano armada, extorsiones, micrográfico, entre otros; se pretende dar a conocer las diferentes medidas de seguridad que pueden adoptar la planta de docentes de los dos colegios de Ciudad Bolívar por medio de la implementación de rutas seguras para la prevención en los casos que el profesorado pueda ser víctima de alguno de los factores del riesgo público y así evitar lesiones físicas o daños psicológicos por este tipo eventos.

Cuando se realizaron las encuestas a los docentes se evidenció en enlace con los resultados obtenidos y que se muestran en las figuras anteriores, que los docentes de estos dos centros se sienten en estado de amenaza constante, puesto que en estos resultados los docentes frente al tema de transporte indican que SI se han presen- tado o evidenciado robos o atracos en el servicios público, es decir, mientras realizan los recorridos para llegar o salir de su sitio de trabajo que involucre las rutas y que estos eventos se presentan con mayor frecuencia en la jornada de la tarde; además de que en medio de las encuestas y las entrevistas también se realiza el sondeo sobre si los docentes conocen o identifican CAI fijos o móviles para realizar reportes de ser necesario, a lo cual un $63 \%$ de los encuestados nos corroboran que no conocen de estos puntos y por ende no saben a dónde acudir para sentirse seguros y denunciar los posibles casos de riesgo a los que se encuentran expuestos diariamente para ejercer su labor de docencia en estas entidades.

Adicional, si se toma en cuenta el conocimiento por parte del profesorado frente a este tema, también se evidenció que los docentes desconocen los factores de riesgo público a los que pueden estar expuestos; es decir en relación con el significado del riesgo público que se encuentra en el DECRETO 423 DE 2006, art. 3; los docentes no reconocen todos los aspectos de estos eventos como afectaciones por este tipo de riesgo, lo cual conlleva a la no notificación oportuna y veraz de los eventos a las entidades pertinentes. A pesar de ello, y por otro lado los docentes sí reconocen casos específicos como lo son los casos de hurto en las proximidades de los planteles, siendo algunos de ellos víctimas de estos.

Abortando el tema puntual del cual trata este artículo que son las rutas seguras, los profesores o docentes de estas instituciones no evidencian rutas seguras para el desplazamiento al lugar de trabajo en este caso la institución educativa y viceversa. Existe un desconocimiento tanto por los educadores como de otros colaboradores sobre los Centros de Atención Inmediata CAI de la Policía Nacional cercanos a los colegios punto que se trata en los entregables que se realizaron como alternativa de prevención a este riesgo.

Por ello es importante dar a conocer las alternativas que ayudan a minimizar estos eventos, para que los docentes no sean foco de agresiones, es en sí que este artículo es una alternativa de ruta segura para estas dos entidades educativas, y que junto con la comunidad ayuden a que estos docentes que realizan su aporte en conocimiento a los hijos de esta comunidad se sientan protegidos por ella y puedan realizar su desplazamiento de una manera más tranquila a la habitual 


\section{Referencias bibliográficas}

Acosta, H. B. (2015). Entorno y posibilidades del ejercicio profesional y sindical de los docentes en Colombia. Educación y violencia, 45-61. https://dialnet.unirioja.es/servlet/articulo?codigo=5474255

Alcaldía Mayor de Bogotá D.C. (2007). Decreto 563. "Plan Maestro de Equiámientos de Seguridad Ciudadana,Defensa y Justicia para Bogotá D.C. http://www.alcaldiabogota.gov.co/sisjur/normas/Norma1.jsp?i=27753

Alcaldía Mayor de Bogotá. (2016). Plan Distrital de Desarrollo 2016-2012: Bogotá mejor para todos. Bogotá, Colombia. http://veeduriadistrital.gov.co/sites/default/files/Sector\%20Planeacion\%20en\%20PDD\%20-\%20SDP.pdf

Alcazar, J. A. (2008). El Perfil Docente: Profesión De Riesgo. Federación De Enseñanza, 1-6. Obtenido De Temas Para La Educación: https://www.feandalucia.ccoo.es/docu/p5sd6251.pdf

Andalucía, F. d. (SF de 09 de 2012). El Malestar Docente. Temas Para La Educacion, págs. 1-6. https://www.feandalucia. ccoo.es/andalucia/docu/p5sd9615.pdf

Amaya, L. (2010). Seguridad y Participación Ciudadana. Obtenido de http://elfaro.net/es/201010/opinion/2669/Seguridady-participaci\% $3 \%$ B3n-ciudadana.htm

Ávila, A. P. (2011). Mercados de criminalidad en Bogotá. Colombia: Taller de Edición-Rocca.

Bogotá, R. (24 de 04 de 2013). Colegios: inseguros adentro y afuera. Obtenido de EL ESPECTADOR: https://www. elespectador.com/noticias/bogota/colegios-inseguros-adentro-y-afuera-articulo-418247

Botero, J. C. (07 de 04 de 2016). Conceptualización sobre pobreza, desigualdad y exclusión social. Obtenido de Desarrollo Social Contemporáneo: http://aulas.uniminuto.edu/campus/pregrado/file.php/2648/Conceptualizacion\%20sobre\%20 pobreza\%2C\%2odesigualdad\%20y\%20exclusion\%2osocial.pdf

Cepeda-Cuervo E, Moncada-Sánchez E, Álvarez V. Violencia intrafamiliar que afecta a estudiantes de educación básica y media en Bogotá. Rev Salud Pública. 2007; 9(4):516-28. http://www.redalyc.org/pdf/422/42219060004.pdf

Cepeda E, Fernández H, Niño V, Navarro V. Factores asociables al logro cognitivo: referente teórico. Colombia: Ministerio de Educación Nacional (Informe técnico); 1997. http://unesdoc.unesco.org/images/o018/001867/186769S.pdf

Cleroux, M. J. (1993). Realización e interpretación de los estudios descriptivos. En M. J. Cleroux, Epidemiología, principios y técnicas (págs. 77-96). Barcelona: Masson Salvat. http://www.psicothema.com/pdf/687.pdf

Cordeiro J, Guillén C, Gala F. Educación primaria y síndrome de burnout: situación de riesgo en los y las docentes de la Bahía de Cádiz [Internet]. Cádiz: Universidad de Cádiz; 2001 [Citado: 2008 Sep 14]. Disponible en: www.stes.es/salud/ Libro_Riesgos_laborales/co5a1.pdf.

De la Cruz, M. A., Grad, H.M. y Hernández E. (1990). Formación pedagógica de los profesores de la Universidad Autónoma de Madrid. Creación de servicio de ayuda a la docencia universitaria. Actas II Jornadas nacionales de didáctica universitaria. file://C:/Users/leherreramar/Downloads/Formaci\%C3\%B3n\%20N7y8.pdf

Desveaux, Y. (o3 de 20 de 2015). Fortalecimiento a organizaciones sociales y comunales. Obtenido de universidad EAFIT: http://www.eafit.edu.co/social/proyectos/Paginas/fortalecimiento-a-organizaciones-sociales-y-comunales.aspx

Escamilla, M. D. (2018). Unidad 3. Aplicación Básica en los métodos científicos. Obtenido de Asignatura de fundamentos de la metodología: https://www.uaeh.edu.mx/docencia/VI_Presentaciones/licenciatura_en_mercadotecnia/fundamentos_ de_metodologia_investigacion/PRES39.pdf

Espectador, E. (29 de 07 de 2012). Docentes en riesgo. Obtenido de EL ESPECTADOR: https://www.elespectador.com/ opinion/docentes-en-riesgo-columna-363675

Fernández M., Desgaste psíquico burnout- en profesores de educación primaria Lima metropolitana. Revista Facultad de Psicología. 2002; 4(5):29-66. https://revistas.ulima.edu.pe/index.php/Persona/article/viewFile/842/814

García-Córdoba, F. G.-C. (SF de SF de 2005). LA PROBLEMATIZACIÒN. Obtenido de Instituto Superior De Ciencias De La Educaion Del Estado De Mexico: file://C:/Users/leidy\%2oherrea/Documents/leidy\%20unim/8\%2oCUATRIMESTRE/ METODOLOGÍA\%20DE\%20LA\%2OINVESTIGACIÓN/ACT\%202/la-problematizacion\%201.pdf

Garrido, A. M. (12 de 2009). Los Riesgos Laborales De La Enseñanza. Obtenido de Cuadernos de Educación y Desarrollo: http://www.eumed.net/rev/ced/10/amg.htm 
Gómez Durán, E. L., Arimany Manso, J., \& Gómez-Alarcón, M. (2012). Las Agresiones a profesionales sanitarios. ESPAÑA: Revista española de medicina legal: órgano de la Asociación Nacional de Médicos Forenses, 2012, Volumen 38, Número 1. http://www.elsevier.es/es-revista-revista-espanola-medicina-legal-285-articulo-las-agresiones-profesionalessanitarios-So377473212000119

Gómez-Restrepo, C., Padilla M., A., Rodríguez, V., Guzmán, J., \& Mejía, G. (2010). Influencia de la violencia en el medio escolar y en sus docentes: estudio en una localidad de Bogotá, Bogotá, D.C., Colombia: Asociación Colombiana de Psiquiatría. http://www.redalyc.org/articulo.oa?id=80615449004

González Placencia, L., Álvarez, M., \& Arce, J. L. (2009). Inseguridad: perspectivas desde América Latina. America Latina: Editorial Miguel Ángel Porrúa. http://www.gandhi.com.mx/inseguridad-perspectivas-desde-america-latina-1

Guerrero E. Análisis pormenorizado de grados de burnout y técnicas de afrontamiento Del estrés docente en profesorado universitario. Anales de Psicología de España. 2003; 19(1):145-58. http://www.um.es/analesps/v19/v19_1/14-19_1.pdf

Hernandez R. (2014). Metodología de la Investigación. Pág. 7-93. México DF: INTERAMERICANA EDITORES S.A. http:// observatorio.epacartagena.gov.co/wp-content/uploads/2017/o8/metodologia-de-la-investigacion-sexta-edicion. compressed.pdf

Jimenez, L. A. (2006). En el país, hay 605 profesores de colegios oficiales amenazados. EL TIEMPO, 1. http://www.eltiempo. com/archivo/documento/CMS-16650066

López, M., Rivera, E., \& Herencia, M. (sf de 09 de 2017). Educación Física Y Desigualdad Social. Percepciones De Los Docentes En Centros De Riesgo De Exclusión Social En La Zona Norte De La Provincia De Granada. Edited by: D.A.A. Scientific Section Martos (Spain), 53-64. Obtenido de Journal of Sport and Health Research: http://www.journalshr. com/papers/Vol\%209_N\%201/Vo9_1_5.pdf

Malamud, A. F. (2012). Inseguridad y violencia en la educación. Problemas y alternativas. Perfiles educativos, SD. http:// www.scielo.org.mx/scielo.php?pid=S0185-26982012000500011\&script=sci_abstract

Medellín, A. d. (13 de 06 de 2013). Alcaldía de Medellín. Obtenido de MANUAL DE BIOSEGURIDAD 2013: http://aulas. uniminuto.edu/campus/mdl_201650/pluginfile.php/40701/mod_resource/content/1/Anexo\%2013.\%20Manual\%20 de\%2obioseguridad\%20de\%2ola\%2oAlcald\%C3\%ADa\%20de\%2oMedell\%C3\%ADn.pdf

Mendigaña, G. G. (05 de 05 de 2017). Seguridad de los empleados: El riesgo público. Obtenido de Riesgo público, factor importante en la seguridad de los trabajadores: http://ccs.org.co/salaprensa/index.php?option=com_content\&view=arti cle\&id=762:riesgo-publico\&catid=361:noticias-agosto-2016\&Itemid $=899$

Murcia, L. M. (2016). ¿Para qué la DE-construcción de la Educación para el Desarrollo? Madrid: Extraído de la charla pronunciada en el centro cultural. http://www.academia.edu/8495588/Para_qu\%C3\%A9_la_DE-construcci\%C3\%B3n_ de_la_Educaci\%C3\%B3n_para_el_Desarrollo

Noguera Zeledón, Z. E. (07 de 03 de 2017). Factores que inciden en la disciplina laboral del personal docente y administrativo que atiende la educación primaria del Colegio Público Las Américas, Barrio Américas 2, localizado en el distrito VI de la ciudad Managua, departamento de Managua durante e. Obtenido de REPOSITORIO UNAN: http:// repositorio.unan.edu.ni/id/eprint/3566

Ospina, L. V. (19 de 02 de 2013). ¿Por qué se asesina a los maestros en Colombia? Obtenido de ARCOIRIS: https://www. arcoiris.com.co/2013/o2/por-que-se-asesina-a-los-maestros-en-colombia/

Parra, V., Guzmán, L., Caldas, M., \& Castro, C. (21 de 04 de 2013). Riesgos en el trabajo. Obtenido de Orden, Aseo Y Practicas De Trabajo Seguro En El Ambiente Laboral: http://riesgoseneltrabajo-38o-b.blogspot.com.co/2013/o4/ordenaseo-y-practicas-de-trabajo_2589.html

Padilla A, Gómez-Restrepo C. Modelo de Grupos de Trabajo en Resolución de Conflictos (GTRC). Curso de Basic Needs Colombia, Experiencia en la Localidad de Usaquén. Conferencia Ministerio de Educación Nacional; 2009 http://www. redalyc.org/pdf/806/80615448013.pdf

Publico, P. (13 de 06 de 2012). Ser docente se convirtió en una profesión de alto riesgo. Obtenido de PERIODISMO PUBLICO: http://www.periodismopublico.com/Ser-docente-se-convirtio-en-una

Rabadà, I. (2006). Identificación de los factores de riesgo laboral en docentes: un estudio Delphi. En I. y. Rabadà, Identificación de los factores de riesgo laboral en docentes: un estudio Delphi (págs. 53-61). sd: Arch Prev Riesgos Labor 2002. http://www.archivosdeprevencion.com/view_document.php?tpd=2\&i=1291 
RCN, N. (20 de 02 de 2018). Alerta De Las Autoridades Por Asesinatos Y Amenazas Contra Profesores. Obtenido De Noticias Ren: http://www.noticiasrcn.com/nacional-pais/alerta-las-autoridades-asesinatos-y-amenazas-contraprofesores

Restrepo-Ayala NC, Colorado-Vargas GO, Cabrera-Arana GA. Desgaste emocional en docentes oficiales de Medellín: Colombia, 2005. Rev Salud Pública. 2006; 8(1):63-73 http://www.scielo.org.co/scielo.php?script=sci_ arttext\&pid=So124-00642006000100006

Rodríguez Mesa, R. (2013). Sistema general de riesgos laborales. ESPAÑA: Universidad del Norte. https://www. librosyeditores.com/tiendalemoine/derecho/3119-sistema-general-riesgos-laborales-ley-1562-2012-reforma-sistemageneral-riesgos-laborales-decreto-723-2013-9789587413595.html

Rodríguez, S. (2015). Educación, Investigación Y Desarrollo Social. Revista Inclusión \& Desarrollo.Sistema Nacional De Bibliotecas Rafael García Herreros, 83-86. http://www.redalyc.org/pdf/310/31044046007.pdf

Rodriguez, H. (2012). Importancia de la formación de los docentes en las instituciones educativas. Recuperado de: https:// www.uaeh.edu.mx/scige/boletin/huejutla/ng/e2.html

Ronderos, M. T. (13 de 03 de 2010). ¿Por qué Colombia no sale del club de los pobres? Obtenido de Semana: http://www. semana.com/nacion/articulo/por-que-colombia-no-sale-del-club-pobres/114313-3

Secretaría Jurídica Distrital de la Alcaldía Mayor de Bogotá D.C.; 11 de Noviembre de 2006; Artículo 3 (Numeral 1); DECRETO 423 DE 2006; "Por el cual se adopta el Plan Distrital para la Prevención y Atención de Emergencias para Bogotá D.C.”. http://www.alcaldiabogota.gov.co/sisjur/normas/Norma1.jsp?i=21916

Sierra Bravo, R. (1995). Técnicas de investigación social. Madrid: Editorial Paraninfo http://www.tirant.com/libreria/libro/ tecnicas-de-investigacion-social-teoria-y-restituto-sierra-bravo-9788428324298

Soriano, J. P. (2009). Seguridad y salud en los docentes. CULTURA PREVENTIVA, 1-6. http://www.intersindical.es/boletin/ laintersindical_saludlaboral_07/archivos/prevenciondocente.pdf

Subias, E. (SF de SF de 2018). Educar en y con seguridad, objetivo del personal docente. Obtenido de Centro de Comunicación y Pedagogía: http://www.centrocp.com/educar-en-y-con-seguridad-objetivo-del-personal-docente/

Tiempo, R. e. (27 de 11 de 2011). Por amenazas, 171 maestros pidieron traslado de colegio. Obtenido de REDACCIÓN EL TIEMPO: http://www.eltiempo.com/archivo/documento/CMS-10843130

Ursua, M. P. (02 de 2012). Miedos comunes en los docentes. Obtenido de CRITICA: http://www.revista-critica.com/larevista/monografico/enfoque/449-miedos-comunes-en-los-docentes

Villaroel, N. C., Lira, J. C., Vergara, C. C., \& Aravena, M. B. (2015). Los Factores De Riesgo Psicosocial Del Trabajo Afectan La Salud Mental De Los Profesores Según El Tipo De Financiamiento Del Establecimiento. Salud \& Sociedad, 1-26. http:// revistas.ucn.cl/index.php/saludysociedad/article/view/938 
Revista incluida en los siguientes agregadores de contenidos

Revista incluida en los siguientes directorios

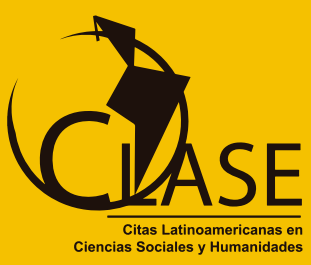

แlbiblat

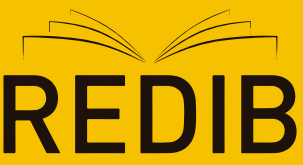

Red Iberoamericana

de Innovación y Conocimiento Científico

\section{Google Académico}

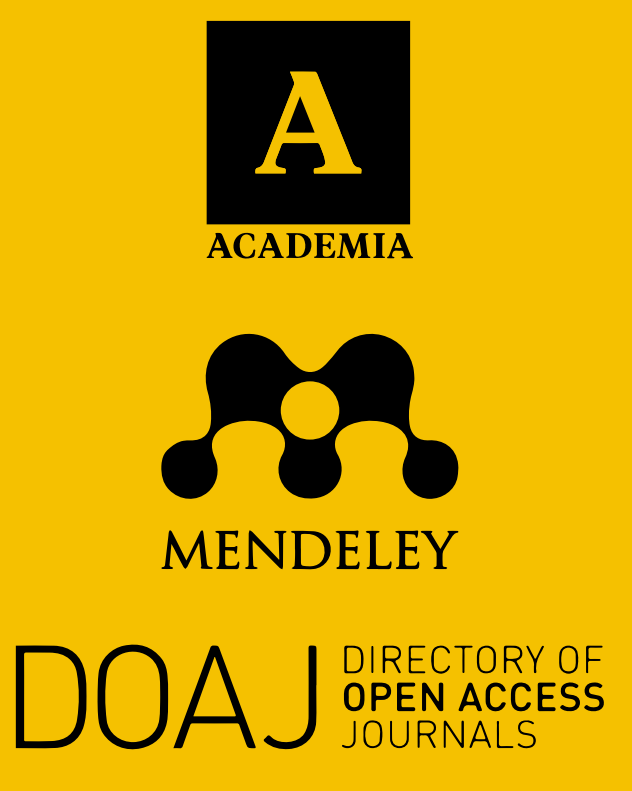

Revista incluida en la siguiente red social

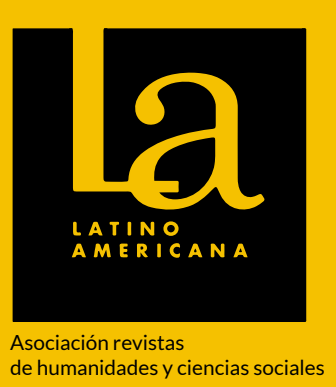



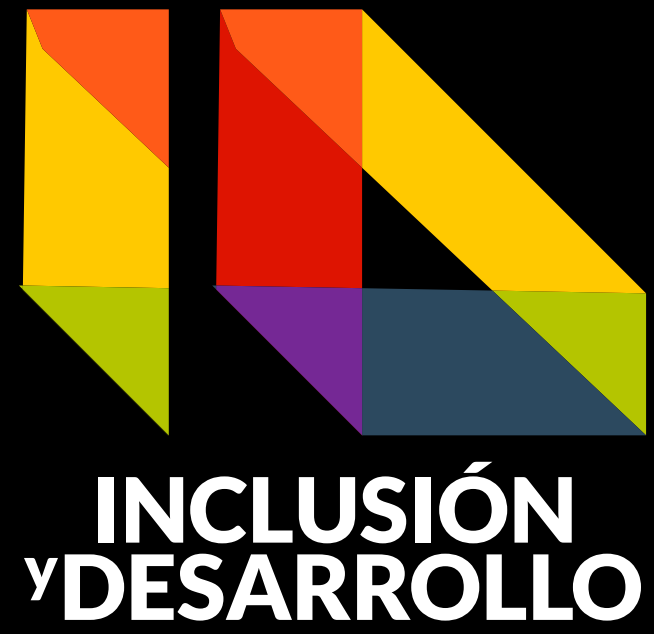

No. 2 Vol. 5 Año 2018 ISSN En línea: 2590-7700

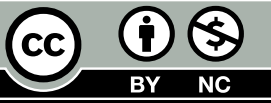

